\title{
A Review of the Clinical Relevance of the Advice and Guidance System Dearbhla McEleney, Shadaba Ahmed
}

An increase in the number of referrals and thus waiting times for appointments in secondary care is an on-going pressure on the NHS. Electronic consultations have been introduced over the years and have expanded to address the challenge of accessing specialist opinions in a secondary care environment. However, it can be a costly service for patients to organise 'e-consultations' themselves, as the majority of these services are only available privately. Advice and Guidance (A\&G) is a relatively new system introduced to the University Hospitals of Morecambe Bay and North Lancashire Clinical Commissioning Groups, to support Primary Care clinicians with referrals and the management of their patients. It enables two clinicians, from primary and secondary care, to communicate via a secure system, seeking advice and support on the management of a patient. Ultimately the process aims to improve patient care whilst reducing the number of referrals to secondary care. This article aims to review the use of the system in the clinical setting and outcomes achieved in the ENT department.

\section{INTRODUCTION}

With advancements in technology, more and more patients opt for telephone consultations to avoid absences from work. More recently, "Electronic Consultations" have been evolving in a bid to provide cost-effective patient care. Applications for smart phones have been introduced by companies, offering private consultations with GPs. However for some patients, paying privately is not an option.

The introduction of an electronic system called Advice and Guidance (A\&G) has proven a success over the past year in the University Hospitals of Morecambe Bay NHS Foundation Trust (UHMB). The system was introduced offering a secure platform to facilitate communication between clinicians in the primary and secondary care environments regarding patient care. With the intention to encourage primary care providers to utilise the system, a simplistic design similar to a text messaging conversation was introduced. A\&G aims to enable primary care to swiftly seek the best clinical pathway or management for their patients to reduce the risk of inappropriate referrals and in turn prevent delay in the treatment of a patient. Primary Care have the option of requesting a telephone call

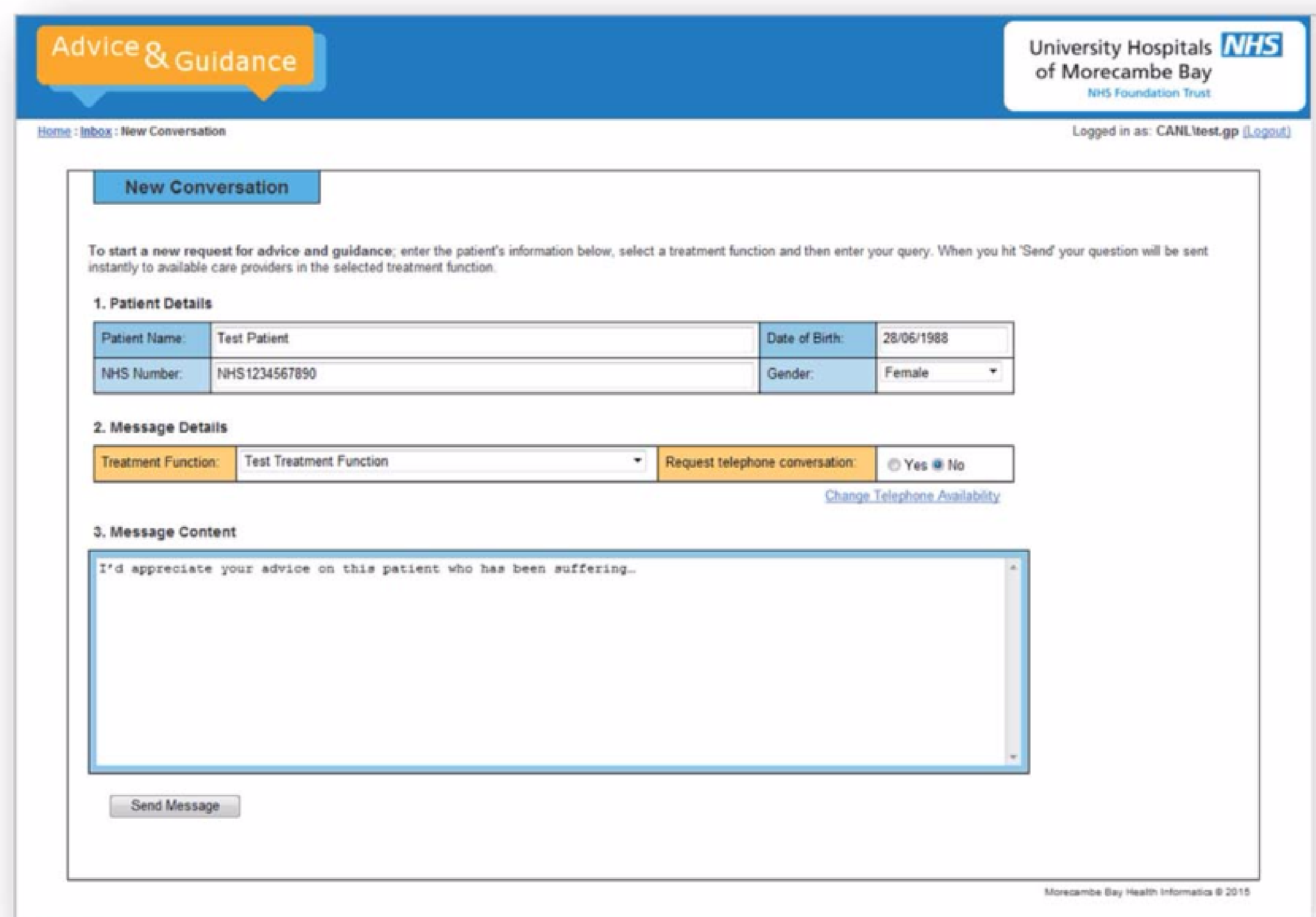


with the secondary care clinician if necessary. This option is useful for complex cases that may be difficult to portray electronically.

The advantage that the $A \& G$ system offered was the ability to add the 'e-conversations' to the patient's permanent clinical records, which can be useful for future consultations.

UHMB piloted this system at the start of 2014 and since then it has been introduced to many specialities including radiology, cardiology, clinical haematology, diabetes/endocrinology, gastroenterology, rheumatology, respiratory, paediatrics and biochemistry. The ENT departments in UHMB piloted this system in February 2015 and have continued to utilize it to date (November 2015). The clinical benefits of this system in ENT and the future of electronic consultations are explored throughout the article.

\section{METHOD}

The A\&G conversations were collected between February and November 2015 and reviewed. The data was entered into Excel and pie charts were produced of the results.

-PUBMED was searched using the terms, cypermedicine, electronic Consultations.

The UHMB trust have an online dashboard that contains the feedback from Primary care, this data was accessed and reviewed.

\section{RESULTS}

Between 3rd March 2015 and 16th November 2015, 38 'conversations' requesting advice were received in the ENT department (36 of which data had been received).

Feedback from primary care clinicians stated that $69 \%$ (pie chart 1) of these cases would have been referred to the outpatient department if they didn't have access to A\&G.

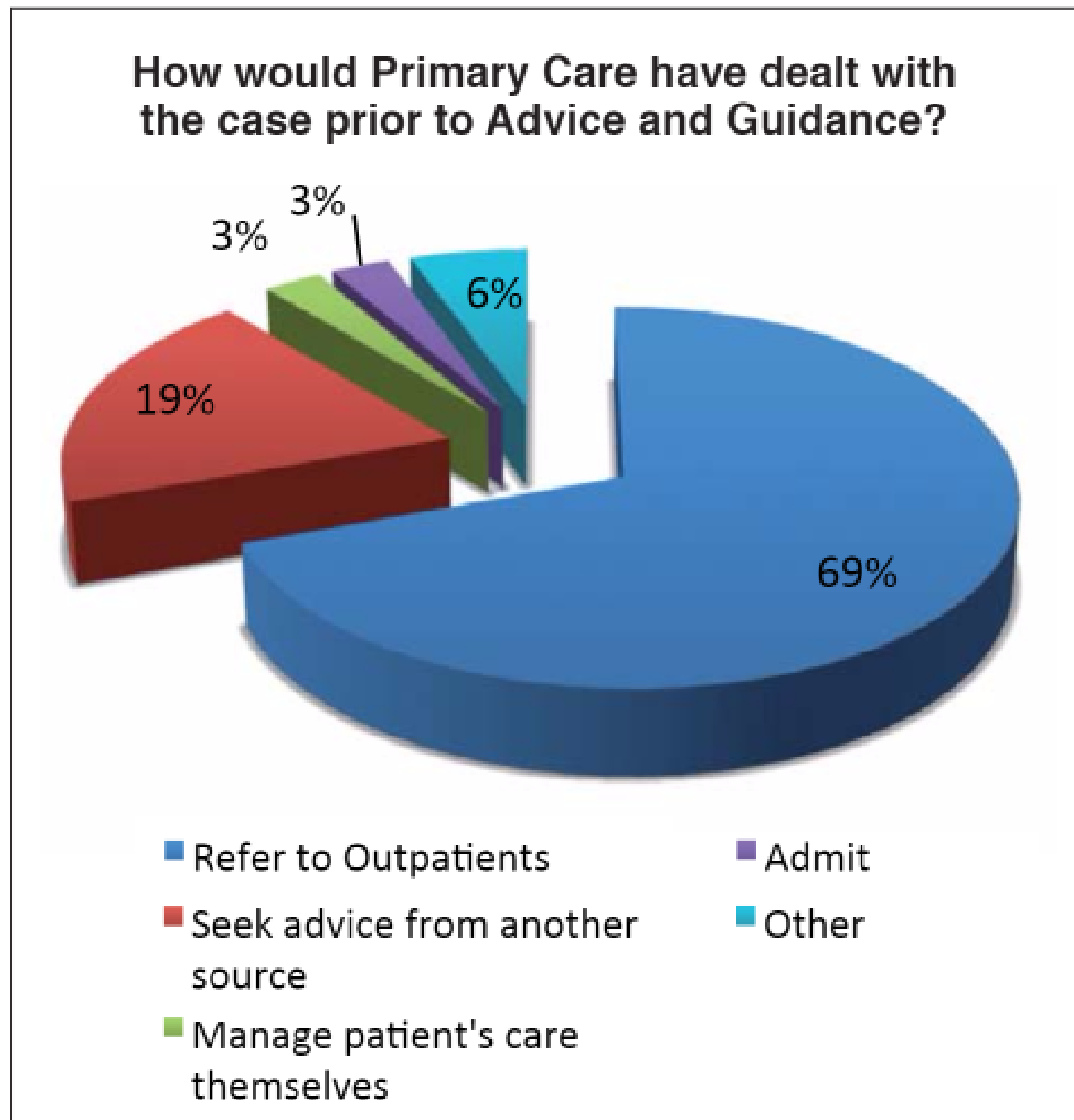

Pie chart 1
Out of the 25 cases that would have initially been referred to outpatients, 18 were referred after using $A \& G$, preventing 7 unnecessary referrals. Only $3 \%$ of the referring clinicians felt they could have dealt with the patients' care themselves, however, after receiving the support from $A \& G$, this figure increased to $17 \%$ (pie chart 1 and 2 ).

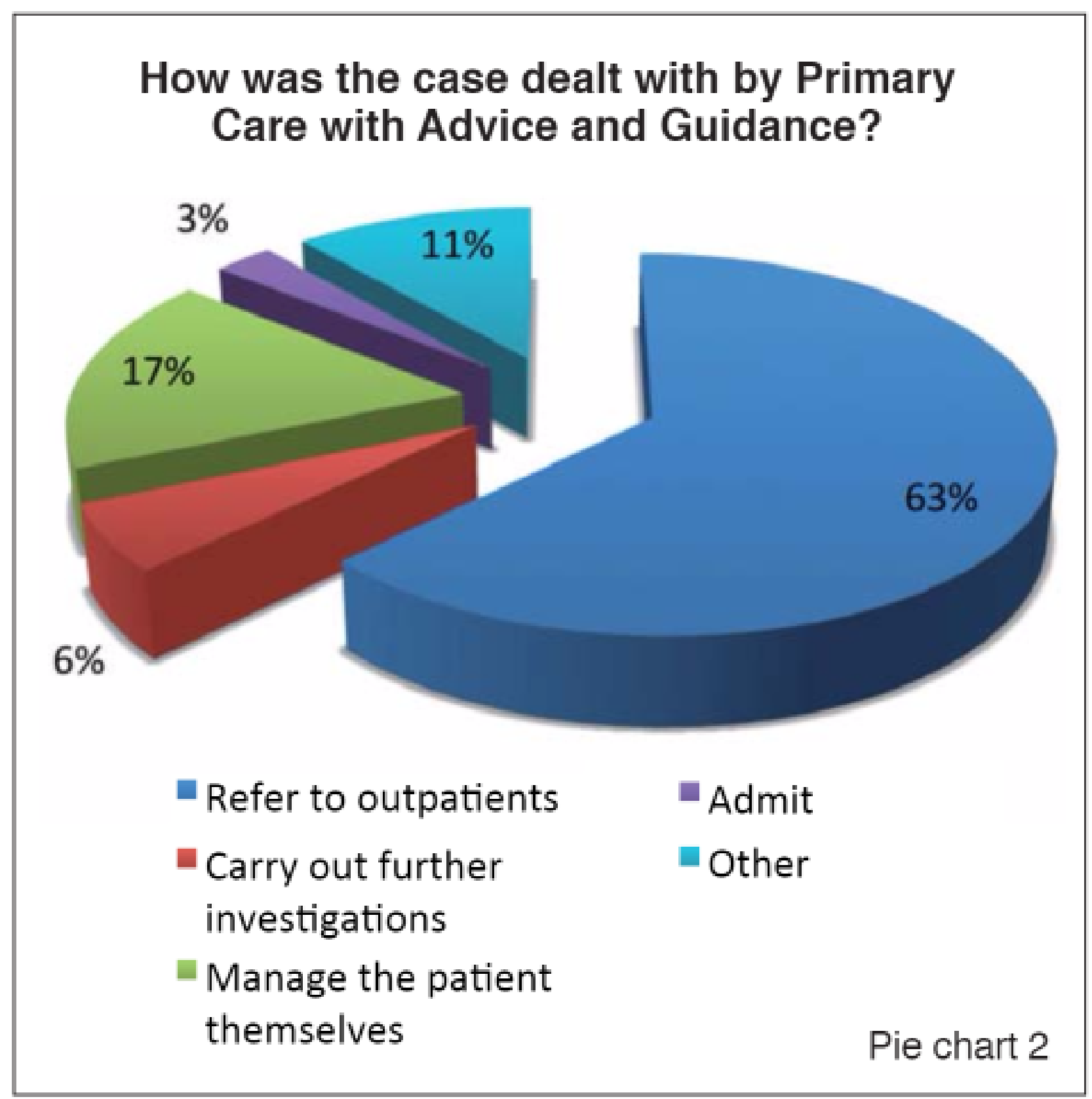

The A\&G system is set up to encourage secondary clinicians to respond to primary care with a rapid response. If a response has not been achieved within 5 days, an automatic reminder is sent to all lead clinicians in the department to flag the need for a response (pie chart 3). The response rate for the ENT department has been very prompt with $68 \%$ of cases receiving a response on the same day, some of which received a response within a few minutes.

\section{Number of Days Taken to Respond}

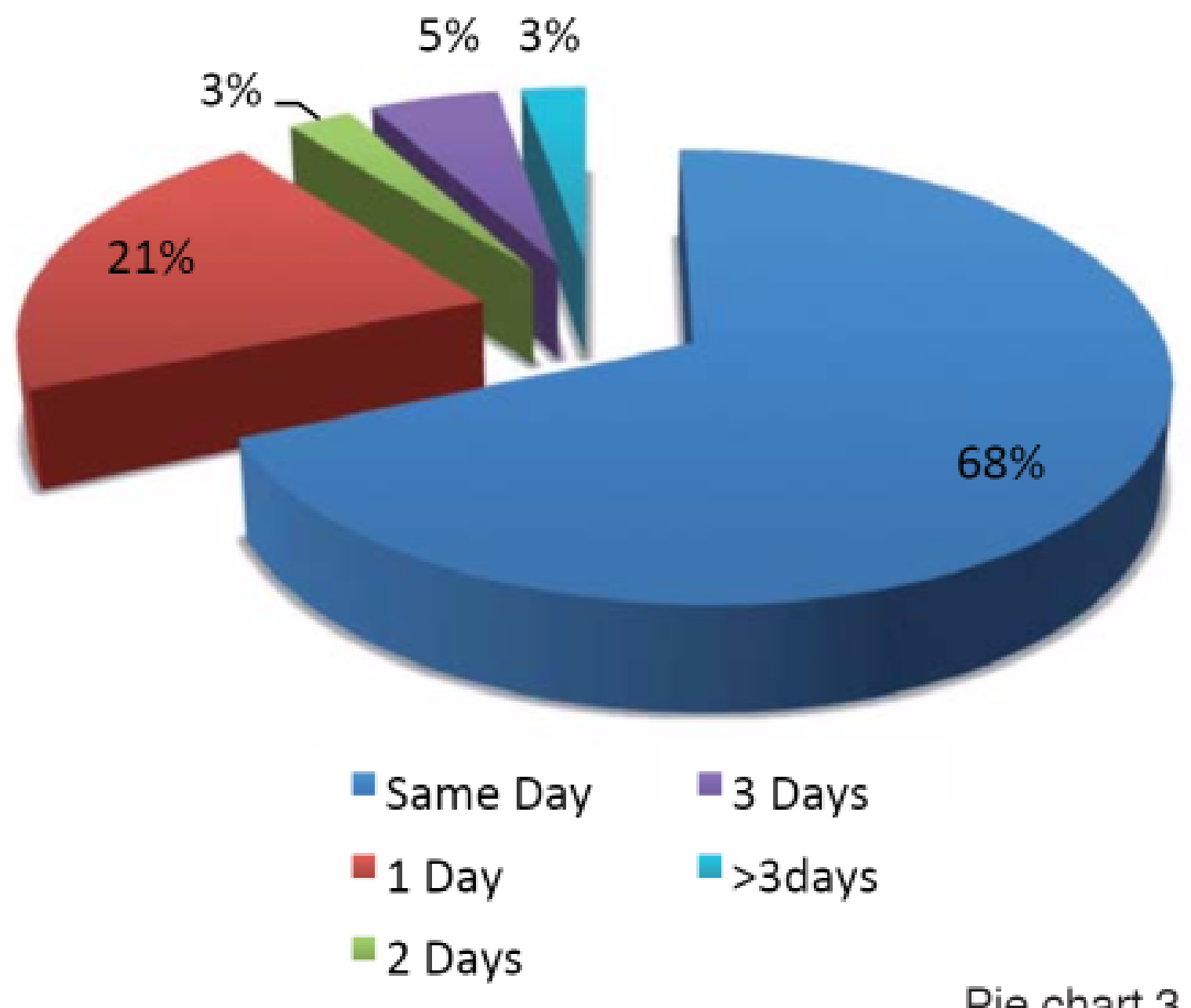


The consensus from Primary Care regarding the accessibility and ease of use of A\&G to the ENT department has been extremely positive. Primary Care satisfaction with the advice given by secondary care has also been encouraging, with $97 \%$ of clinicians finding the service useful (pie chart 4 ).

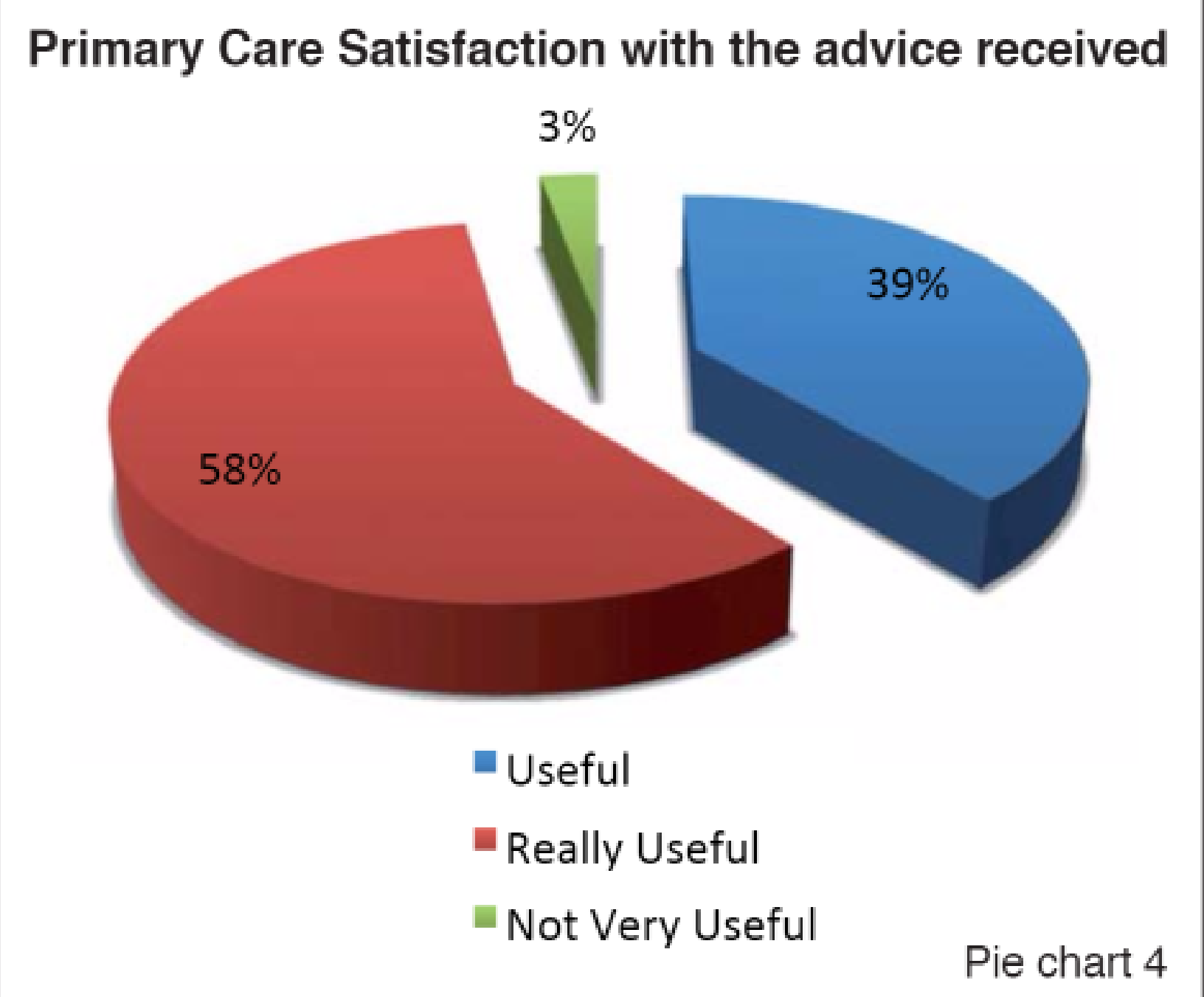

It is evident from the results that the $A \& G$ system is a cost-effective tool that can instil confidence in the primary care community to initiate treatment that they wouldn't have initially considered. This prevents a delay in patient treatment and an increase in patient satisfaction, furthermore, saving time and resources in the primary and secondary care facilities.

\section{DISCUSSION}

Some specialties in UHMB have been inundated with "e-conversations," through A\&G, such as haematology and gastroenterology with over 600 cases per specialty, in comparison to 38 within the ENT department. A systematic review on e-consultations has shown that haematology and endocrinology are among the top specialties requiring e-consults. ${ }^{1}$ This could suggest that primary care clinicians are more comfortable managing ENT cases, in comparison to other specialist areas. This highlights the potential advantage of $A \& G$ as a system that not only addresses the needs of the patients but also benefits the requesting clinician as an educational resource.

However, there are potential pitfalls with such a high volume of case queries to each specialty. E-consultations run the risk of communication failure, such as misinterpretation of information resulting in incorrect diagnosis and therefore delayed treatment. It can also run the risk of unnecessary advice being sought from specialists when the patient should have been referred. For example some of the cases discussed in the A\&G in ENT were cases that should have been referred under urgent 2-week referral times. Finally, if primary care clinicians become overly reliant on the $A \& G$ service, whilst reducing the number of referrals to the outpatient department, they may become a burden on the specialists, as in some cases it takes time to think about cases and find information prior to responding.

Currently there are private "e-consultations" available in the UK, with one of the newest websites, 'Babylon' which offers a consultation application for smart phones. This website offers individuals to pay a monthly subscription or one off payment to book a video or telephone consultations with a GP or a specialist. ${ }^{2}$ It offers patients the option of asking one question to GP and attaching an image if necessary, however, it only permits one question to be sent and no further conversation, unless a consultation is requested. ${ }^{2}$ Over 250,000 people in the UK and Ireland are utilizing this service. ${ }^{3}$ Although this service is available on the NHS for patients registered in two GP practices in Essex, not everyone can afford this service privately. ${ }^{2}$ However, the issues that may arise from using these services involve having consultations with patients without having access to their medical records. In some instances patients exclude information that they deem irrelevant which may in fact be vital for a consultation. A\&G doesn't offer the service of communication between the patient and primary care clinicians. However, the future for A\&G looks promising with the hope of creating a "Patient Room", where the e-consultation will soon allow the patient to be introduced to the discussion between clinicians. This will reduce the risks of dissatisfied patients who were hoping to see a secondary care clinician and who dislike the idea of being managed by primary care clinicians who aren't specialists in particular fields. The idea of being involved in the discussions and management pathways has the potential to improve patient satisfaction.

\section{CONCLUSION}

The data for A\&G within the ENT department is a relatively small caseload in comparison to other specialties, it has however, proven that $A \& G$ service is beneficial for the patients and clinicians involved. At present images can't be added to the A\&G system but future developments show potential for the images to be added. It is however extremely beneficial that $A \& G$ is attached to patient records therefore scans and investigation results can be accessed by secondary care providers in order to provide the best possible care for the patient. Furthermore, the aforementioned potential of introducing a "patient room" opens exciting doors for the development of e-consultations in UHMB, a system which could be utilized in many other trusts.

\section{REFERENCES}

1. Vimalananda VG, Gupte G, Seraj SM, Orlander J, Berlowitz D, Fincke BG, et al. Electronic consultations (e-consults) to improve access to specialty care: a systematic review and narrative synthesis. Journal of telemedicine and telecare. 2015 Sep;21(6):323-30. PubMed PMID: 25995331. Pubmed Central PMCID: 4561452 .

2. Babylon. [24th November 2015]. Available from: http://www.babylonhealth.com.

3. BBC News. How mobile devices are changing healthcare [24th November 2015]. Available from: http://www.bbc.co.uk/news/business-34900169.

Correspondence to: Shadaba Ahmed Consultant Ear, Noise \& Throat Surgeon, RLI 